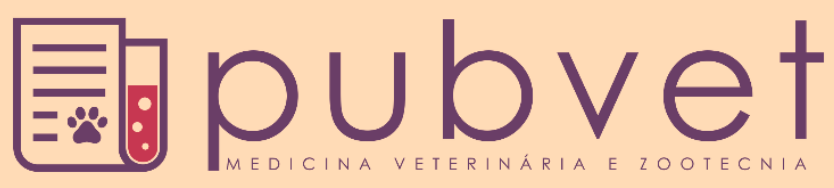

https://doi.org/10.31533/pubvet.v14n10a667.1-5

\title{
Abordagem cirúrgica da síndrome da dilatação volvo gástrica em um cão: Relato de caso
}

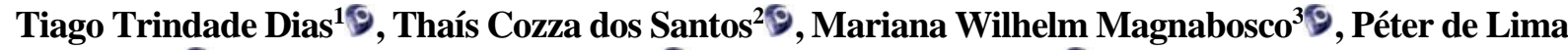

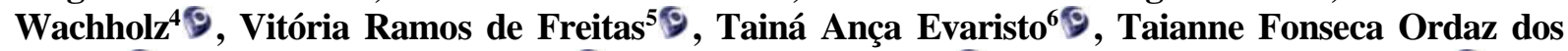

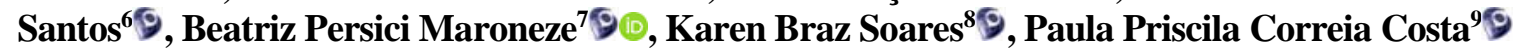 \\ ${ }^{1}$ Médico Veterinário, Universidade da Região da Campanha. *Autor para correspondência, E-mail: tiagotdias@hotmail.com \\ ${ }^{2}$ Médica Veterinária, Mestranda em Programa de Pós-graduação em Medicina Veterinária, Universidade Federal de Pelotas (UFPel), Brasil. \\ ${ }^{3}$ Médica Veterinária, Pós-graduanda em Diagnóstico por Imagem, Residência Multiprofissional em Área da Saúde, UFPe \\ ${ }^{4}$ Médico Veterinário, Pós-graduando em Clínica Médica de Animais de Companhia - Residência Multiprofissional em Área da Saúde, UFPel \\ ${ }^{5}$ Médica Veterinária, Pós-graduando em Anestesiologia Veterinária, Residência Multiprofissional em Área da Saúde, UFPel \\ ${ }^{6}$ Discente em Medicina Veternária, UFPel \\ ${ }^{7}$ Médica Veterinária, Mestranda em Programa de Pós-graduação em Medicina Veterinária, Universidade Federal do Rio Grande do Sul \\ ${ }^{8}$ Médica Veterinária, Pós-graduanda em Anestesiologia de Pequenos Animais (IBMVet) \\ ${ }^{9}$ Docente no Departamento de Clínicas Veterinárias, Hospital de Clínicas Veterinárias, UFPel
}

Resumo. A dilatação vólvulo gástrica se instala quando o estômago se enche excessivamente de gás ocasionando uma dilatação, e consequentemente uma rotação do estômago sobre seu eixo mesentérico. A causa desta síndrome é desconhecida, mas exercícios após volumosas refeições favorece a ocorrência desta patologia. Por consequência da distensão seguida de torção gástrica, o estômago comprime a veia cava caudal e veia porta, acarretando em inúmeros distúrbios da circulação que prejudicam diretamente o sistema cardiorrespiratório. Diante deste e de outros fatores a alta taxa de mortalidade é uma realidade, portanto quanto antes o paciente for hospitalizado e diagnosticado, terá mais chances de sobreviver. $\mathrm{O}$ tratamento consiste primeiramente em estabilizar o paciente para depois seguir as metas da intervenção cirúrgico.

Palavras chave: dilatação, estômago, síndrome, torção, vólvulo

\section{Surgical approach of syndrome of gastric dilatation-volvulus in a dog: Case report}

\begin{abstract}
The syndrome of gastric dilatation-volvulus occurs when there is a rapid accumulation of air in the stomach causing a dilatation, consequently the stomach rotates around the mesenteric axis. The cause of this syndrome is still unclear, but dogs that exercise after eating are at a higher risk of developing this pathology. The distention and displacement of the stomach compress the caudal vena cava and portal vein, resulting in several cardiovascular effects that harm directly the cardiorespiratory system. Considering these and other factors, the high mortality rate is a reality, then the sooner the patient is hospitalized and diagnosed, the greater is the chance of surviving. The treatment consists primarily in stabilize the patient and then follow the goals of chirurgic intervention.
\end{abstract}

Keywords: dilatation, stomach, syndrome, volvulus

\section{Abordaje quirúrgico del síndrome de dilatación vólvulo-gástrica en un perro: Relato de caso}

Resumen. La dilatación vólvulo gástrica se instala cuando el estómago se llena excesivamente de gas ocasionando una dilatación, y consecuentemente rotación del 
estómago sobe su eje mesentérico. La causa de este síndrome es desconocida, aunque perros que se ejercitan después de alimentarse tienen mayor riesgo de desarrollar esta patología. Por consecuencia de la distensión seguida de la torsión gástrica, el estómago comprime la vena cava caudal y la vena porta, acarretando varios disturbios de circulación que perjudican directamente el sistema cardiorrespiratorio. Delante de estos y otros factores la alta tasa de mortalidad es una realidad, entonces cuanto antes se hospitalice y diagnostique el paciente, mayores son las posibilidades de sobrevivir. El tratamiento consiste primeramente en estabilizar al paciente y después seguir con las metas de la intervención quirúrgica.

Palabras clave: dilatación, estómago, síndrome, torsión, vólvulo

\section{Introdução}

A síndrome da dilatação volvo gástrica (DVG) é uma enfermidade clínica-cirúrgica de emergência, que envolve diversos fatores fisiopatológicos, onde se não tratados imediatamente poderão culminar com óbito do paciente. Os animais acometidos são, principalmente, caninos de raças grandes e gigantes, no entanto existem relatos de cães de raças pequenas e, gatos, com tal patologia (Silva et al., 2006).

Esta condição trata-se do aumento exacerbado do estômago associado ao seu mau posicionamento, ocasionando um volvo parcial, onde o esvaziamento é barrado devido a uma compressão sobre o piloro e duodeno. Na maioria dos casos a dilatação ocorre antes do volvo (Rasmussen, 2007).

O piloro e o cárdia são bases de referências para determinar o grau de rotação, onde é analisado o desvio do eixo longitudinal que, geralmente enquadra-se entre 270 e 360 graus. É determinada uma "torção" se a rotação for inferior a 180 graus e volvo, quando superior (Rasmussen, 2007).

$\mathrm{O}$ tratamento consiste primeiramente em estabilizar o paciente, reduzindo os sintomas apresentados, para posteriormente tratar de modo cirúrgico as complicações da doença, que variam de necrose da parede gástrica por isquemia à ruptura estomacal, laceração e/ou avulsão da vasculatura esplênica, liberação de microrganismos da lâmina própria intestinal por ausência de oxigenação celular local, até a manifestação de sinais compatíveis com fatores cardiodepressores do pâncreas isquêmico, que ocorre na fase mais grave e, final, do caso, havendo sobreposição dos tipos de choque, evoluindo, em sua maioria, para o insucesso no restabelecimento da condição do animal e, óbito (Aguiar, 2011).

As metas do tratamento cirúrgico consistem em avaliar o estômago e o baço para a ressecção de prováveis tecidos necróticos, aliviar o estômago e posicioná-lo em seu sentido correto e, por último, fixar o estômago à parede abdominal para não ocorrer recidivas (Fossum, 2015).

O presente trabalho visa apresentar um relato de caso do tratamento cirúrgico de uma torção vólvulo gástrica de um cão.

\section{Relato de caso}

Foi atendido no Hospital Dra. Renata Saccaro, localizado em Caxias do Sul, Rio Grande do Sul, um cão, da raça Dogue Alemão, de dez anos de idade, pesando $70 \mathrm{~kg}$.

$\mathrm{Na}$ consulta os tutores relataram que após a alimentação, o paciente se apresentou prostrado, em decúbito lateral com um aumento de volume abdominal.

No exame clínico foi observado abdômen abaulado, som timpânico na percussão, pulso periférico fraco, taquicardia, dispneia, tempo de preenchimento capilar aumentado, mucosas pálidas.

Suspeitando de dilatação gástrica realizou-se radiografias da região abdominal (Figura 1), onde confirmou o diagnóstico de síndrome da dilatação vólvulo gástrica.

Em conseguinte, o paciente foi acessado na veia cefálica dos dois membros torácicos, onde em um dos acessos estava solução de ringer com lactato em taxa de $85 \mathrm{~mL} / \mathrm{kg} / \mathrm{h}$ para a correção hidroeletrolítica na primeira hora e, no outro acesso endovenoso estava sendo administrado ringer lactato suplementado com $5 \mathrm{~mL}$ de cloreto de potássio $(\mathrm{KCl})$ na taxa de $40 \mathrm{~mL} / \mathrm{kg} / 24 \mathrm{~h}$. 
Após, foi realizada a medicação pré-anestésica (MPA) com metadona $(0,3 \mathrm{mg} / \mathrm{kg})$ e midazolam $(0,3$ $\mathrm{mg} / \mathrm{kg}$ ), via intramuscular, dando sequência a tricotomia da região hipogástrica e mesogástrica abdominal.

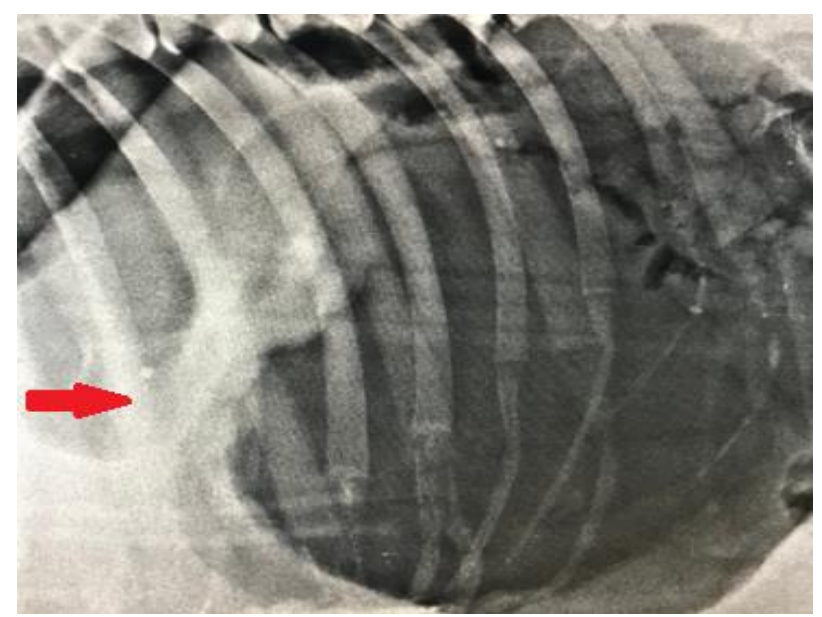

Figura 1. Radiografia latero-lateral abdominal do paciente, demonstrando dilatação gástrica e sinal de compartilhamento.

Após a MPA, foi realizada a trocanterização do estômago, utilizando-se do aparelho de ultrassonografia como guia de referência, tendo como finalidade a descompressão gasosa, bem como alívio imediato ao animal, dada a gravidade da condição.

Em sequência, foi realizada a passagem de uma sonda oro-gástrica para esvaziar o estômago, porém este procedimento não obteve sucesso devido a obstrução na região do cárdia em decorrência da torção gástrica.

Para preparação cirúrgica, foi realizada indução anestésica endovenosa com lidocaína $(1 \mathrm{mg} / \mathrm{kg})$ e propofol $(4 \mathrm{mg} / \mathrm{kg})$. Na manutenção anestésica, manteve-se o paciente com anestesia inalatória, utilizando-se de isoflurano (1,5 CAM), bem como a monitoração constante dos parâmetros cardiorrespiratório, dando início ao procedimento cirúrgico.

A técnica cirúrgica consistiu na incisão pela linha média, de pele e subcutâneo, adentrando-se o abdômen por meio da linha alba, sendo necessária incisão do apêndice xifoide até a região cranial a cicatriz umbilical.

Obteve o acesso à cavidade abdominal localizando o estômago, na região hipogástrica, onde o mesmo encontrava-se rotacionado sobre seu eixo longitudinal, impondo a torção do duodeno e o piloro sobre o esôfago e o cárdia.

Primeiramente foi feita trocanterização intra-abdominal, para posteriormente iniciar $o$ reposicionamento do estômago (Figura 2) na sua localização anatômica, não sendo necessária a realização de gastrectomia parcial devido boa viabilidade do tecido gástrico.

O baço apresentava-se em esplenomegalia, contudo, não foi necessário a realização da esplenectomia total devido à ausência de necrose local.

Foi necessário a realização de gastrostomia para colocação de uma sonda gástrica de Foley (Figura 3), tamanho 20, que foi fixada utilizando-se fio multifilamentar absorvível (poligactina 910; 2-0) na parede estomacal e abdominal. Além disso, foi realizada uma gastropexia na parede abdominal contralateral ao estômago, com a finalidade de evitar recidivas.

A celiorrafia foi realizada com sutura interrompida no padrão sultan, redução do espaço morto com sutura simples interrompida e a dermorrafia com sutura interrompida no padrão wolff, ambos com uso de náilon 2-0 - inabsorvível unifilamentar. A fixação da sonda gástrica foi realizada em sinonímia ao padrão de fio para síntese cavitária, utilizando-se do padrão de sutura sandália romana.

O paciente recebeu alta após cinco dias de internação, com indicação terapêutica à domicílio de carprofeno $(2,2 \mathrm{mg} / \mathrm{kg} / \mathrm{via}$ oral $/ 12-12 \mathrm{~h})$ por 5 dias, sucralfato $(1 \mathrm{~g} / \mathrm{animal} / \mathrm{via}$ oral $/ 8-8 \mathrm{~h})$ por 3 dias, 
ranitidina ( $1 \mathrm{mg} / \mathrm{kg} / \mathrm{via}$ subcutânea $/ 8-8 \mathrm{~h})$ por 6 dias, cefalexina $(15 \mathrm{mg} / \mathrm{kg} / \mathrm{via}$ oral $/ 12-12 \mathrm{~h})$ por 6 dias e metronidazol (15 mg/kg/via oral/12-12h) por 3 dias, alimentação pastosa A/D Hills Diet em menor quantidade e maior frequência, com a recomendação de limpeza do curativo da sonda uma vez por dia, uso obrigatório de roupa cirúrgica, repouso absoluto e retorno em cinco dias para reavaliação e remoção da sonda gástrica.

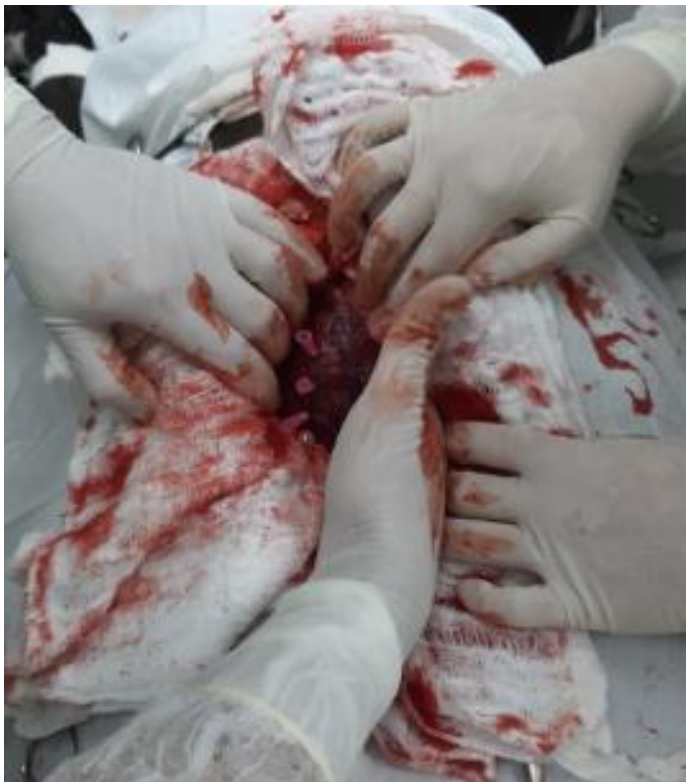

Figura 2. Trocanterização intra-abdominal para remoção da presença de gases gástricos.

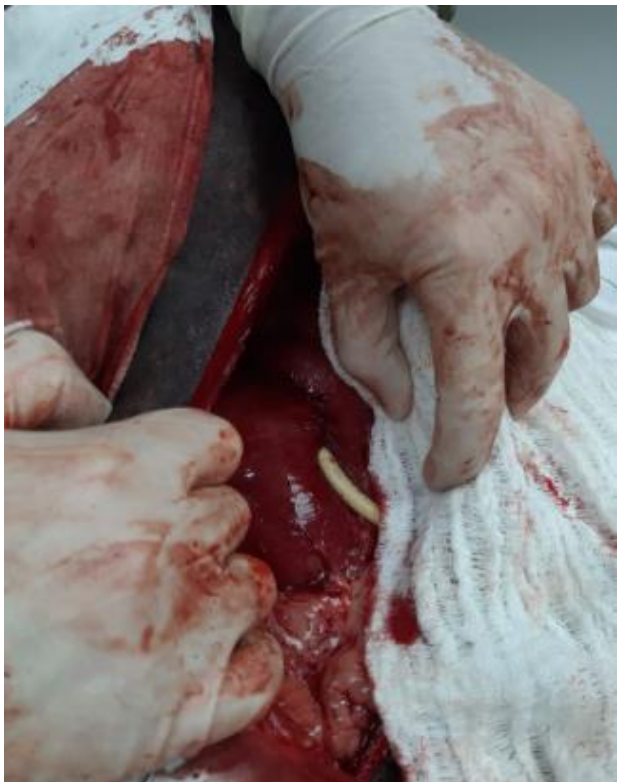

Figura 3. Colocação de sonda gástrica de Foley $\left(n^{\circ} 20\right)$.

\section{Resultados e discussão}

No presente relato, o paciente acometido era um cão, macho, da raça Dogue Alemão, pesando $70 \mathrm{~kg}$, Matthiensen (1996) afirma que a maior incidência de dilatação volvo gástrica está em animais de raças grandes, puras e de tórax profundo, corroborando com os dados do caso. Entretanto, Sturgess (2001) relata a ocorrência dessa síndrome em gatos e cães de raças menores, com maior frequência nos Dachshund, devido ao tórax profundo.

No exame clínico, suspeitou de dilatação vólvulo gástrica por meio de palpação abdominal, por apresentar aumento de volume e a existência de certo grau de timpanismo, no entanto, Fossum (2015) relata que esses são indicativos para suspeitar da dilatação volvo gástrica.

Fossum (2015) ainda menciona a presença de sinais clínicos como: pulso periférico fraco, taquicardia, tempo de preenchimento capilar aumentado, mucosas pálidas e dispneia, assim como no presente relato.

Após a confirmação do diagnóstico, foram feitos dois acessos venosos em ambos membros torácicos do cão e, em seguida a descompressão gástrica com agulhas de diâmetro 40x16 mm, Rasmussen (2007) orienta que seja primeiramente assegurado um acesso venoso para a reposição de fluido, como foi feito no caso descrito, para posteriormente dar sequência a outros procedimentos, como a descompressão gástrica.

Entretanto, Guzman (2010) recomenda a descompressão como primeiro passo para ajudar na eliminação de gases de forma gradativa. Em seguida, com o animal sedado, tentou-se introduzir uma sonda oro-gástrica para remover gases e resto de conteúdo gástricos, Guzman (2010) e Rasmussen (2007) indicam esse procedimento a fim de aumentar a descompressão e associar a lavagem gástrica com água morna, eliminando possíveis microrganismos acumulados na cavidade gástrica.

No momento do acesso a cavidade abdominal constatou-se a rotação do estômago no sentindo horário, assim antes de corrigir esse mau posicionamento foi feito a descompressão com 4 agulhas $40 \mathrm{x}$ $12 \mathrm{~mm}$, seguido da correção, rotacionando o estômago no sentido anti-horário, conforme é preconizado 
por Fossum (2015). Parton et al. (2006) indica a ressecção das áreas necrosadas com gastrectomia parcial, porém neste caso o tecido gástrico não estava necrosado, não sendo necessário esse procedimento.

A gastropexia associada a gastrostomia com sonda de Foley $\mathrm{n}^{\mathrm{o}}$ 20, proporcionou a vantagem de acesso a nutrição enteral, medicamentos via sonda e descompressão gástrica no pós-operatório, estando de acordo com os relatos de Tivers \& Brockman (2009).

\section{Conclusão}

O respectivo relato demostra a importância de diagnosticar e solucionar de modo ágil os sintomas de uma dilatação volvo gástrica, bem como a escolha e execução de abordagens cirúrgicas adequadas a cada caso, aumentando as chances de sucesso no tratamento e reabilitação do paciente.

\section{Referências}

Aguiar, E. S. V. (2011). Emergências decorrentes do trauma em pequenos animais: técnicas e manual para equipes de pronto-atendimento. Editora Da Universidade Federal Do Rio Grande Do Sul (UFRGS), Porto Alegre/RS. $1^{\circ}$ Ed., P., 25-33.

Fossum, T. W. (2015). Cirurgia de pequenos animais. Elsevier Brasil.

Guzman, P. T. (2010). Síndrome dilatación/vólvulo gástrico (DGV). Congreso ecveccs emergencia y cuidados criticos veterinarios.

Matthiensen, D. T. (1996). Fisiopatologia da dilatação gástrica: vólvulo. Bojrab MJ. Mecanismo Da Moléstia Na Cirurgia de Pequenos Animais, 2, 260-273.

Parton, A. T., Volk, S. W., \& Weisse, C. (2006). Gastric ulceration subsequent to partial invagination of the stomach in a dog with gastric dilatation-volvulus. Journal of the American Veterinary Medical Association, 228(12), 1895-1900. https://doi.org/10.2460/javma.228.12.1895

Rasmussen, L. (2007). Estômago. In: SLATTER, D. Manual De Cirurgia De Pequenos Animais., 3, 592-641.

Silva, G. K., Weide, L. A., \& Contesini, E. A. (2006). Síndrome dilatação vólvulo gástrico: fisiopatologia: revisão da literatura. Revta Vet. Em Foco, 3, 119-128.

Sturgess, C. P. (2001). Doenças do trato alimentar. Tratado de Medicina Interna de Pequenos Animais. Ed. Roca, São Paulo, 367-443.

Tivers, M., \& Brockman, D. (2009). Gastric dilation-volvulus syndrome in dogs 1. Pathophysiology, diagnosis and stabilisation. In Practice, 31(2), 66-69. https://doi.org/10.1136/inpract.31.2.66

Recebido: 25 de março, 2020.

Aprovado: 23 de abril, 2020.

Disponível online: 7 de outubro, 2020.
Licenciamento: Este artigoé publicado na modalidade Acesso Aberto sob a licença Creative Commons Atribuição 4.0 (CC-BY 4.0), a qual permite uso irrestrito, distribuição, reprodução em qualquer meio, desde que o autor e a fonte sejam devidamente creditados 